\title{
Quantifying uncertainties of climate signals in chemistry climate models related to the 11-year solar cycle - Part 1: Annual mean response in heating rates, temperature, and ozone
}

\section{Markus Kunze et al.}

Correspondence to: Markus Kunze (markus.kunze@met.fu-berlin.de)

The copyright of individual parts of the supplement might differ from the CC BY 4.0 License. 
Table S1: Solar cycle spectral solar irradiance (SSI) variations for Solar Cycles indicated in the first row relative to ATLAS3 ( $\triangle S S I$ ) in $\%$ and relative contribution of SSI changes to the TSI change $\left(\frac{\Delta S S I}{\Delta T S I}\right)$ in $\%$ for the Lyman- $\alpha(121.5 \mathrm{~nm})$, Far-UV (121-200 nm), Herzberg continuum/Hartley bands (201-242 nm), Hartley-/Huggings-bands (243-380 nm) and visible (381-780 nm) spectral ranges.

\begin{tabular}{|c|c|c|c|c|c|c|c|c|c|c|c|}
\hline Time period & SSI dataset & \multicolumn{2}{|c|}{$\begin{array}{c}121.5 \mathrm{~nm} \\
\Delta S S I \frac{\Delta S S I}{\Delta T S I}\end{array}$} & \multicolumn{2}{|c|}{$\begin{array}{c}121-200 \mathrm{~nm} \\
\Delta S S I \frac{\Delta S S I}{\Delta T S I}\end{array}$} & \multicolumn{2}{|c|}{$\begin{array}{l}201-242 \mathrm{~nm} \\
\Delta S S I \frac{\Delta S S I}{\Delta T S I}\end{array}$} & \multicolumn{2}{|c|}{$\begin{array}{c}243-380 \mathrm{~nm} \\
\Delta S S I \frac{\Delta S S I}{\Delta T S I}\end{array}$} & \multicolumn{2}{|c|}{$\begin{array}{c}381-780 \mathrm{~nm} \\
\Delta S S I \frac{\Delta S S I}{\Delta T S I}\end{array}$} \\
\hline \multirow{5}{*}{$\begin{array}{l}\text { Cycle } 21 \\
\text { descent } \\
\text { Max:Dec.1979 } \\
\text { Min:Sep.1986 }\end{array}$} & 年 & 45.602 & 0.214 & 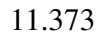 & 0.913 & 3.599 & 4.300 & 0.311 & 20.748 & 0.109 & 55.521 \\
\hline & NRLSSI2 & 51.609 & 0.228 & 11.668 & 0.882 & 3.360 & 3.778 & 0.402 & 25.251 & 0.095 & 45.2 \\
\hline & SATIRE-T & 47.283 & 0.199 & 10.081 & 0.724 & 3.445 & 3.684 & 0.592 & 35.363 & 0.106 & 48.028 \\
\hline & SATIRE-S & 47.178 & 0.281 & 9.907 & 1.008 & 2.987 & 4.523 & 0.477 & 40.368 & 0.069 & 44.130 \\
\hline & CMIP6 & 49.405 & 0.250 & 10.788 & 0.932 & 3.175 & 4.080 & 0.440 & 31.585 & 0.082 & 44.785 \\
\hline \multirow{5}{*}{$\begin{array}{l}\text { Cycle } 22 \\
\text { ascent } \\
\text { Max:Nov.1989 } \\
\text { Min:Sep.1986 }\end{array}$} & I1 & (20. & 0 & - & 1.067 & . & (2) & . & & 0.104 & \\
\hline & NRLSSI2 & 56.657 & 0.273 & 12.808 & 1.053 & 3.668 & 4.488 & 0.407 & 845 & 0.081 & 42.196 \\
\hline & SATIRE-T & 44.322 & 0.247 & 9.454 & 0.899 & 3.235 & 4.580 & 0.529 & 41.801 & 0.075 & 45.107 \\
\hline & SATIRE-S & 59.989 & 0.331 & 12.634 & 1.191 & 3.756 & 5.269 & 0.576 & 45.149 & 0.070 & 41.540 \\
\hline & CMIP6 & 58.338 & 0.300 & 12.722 & & 3.715 & 4.855 & 0.492 & & 0.076 & 42.139 \\
\hline \multirow{5}{*}{$\begin{array}{l}\text { Cycle } 22 \\
\text { descent-2 } \\
\text { Max:Nov.1989 } \\
\text { Min:Nov.1994 }\end{array}$} & NRLSSI1 & 44.286 & 0.266 & 11.067 & 1.137 & 3.482 & 5.324 & 0.268 & 22.913 & 0.084 & 54.902 \\
\hline & NRLSSI2 & 50.377 & 0.291 & 11.388 & 1.125 & 3.257 & 4.788 & 0.354 & 29.039 & 0.066 & 41.415 \\
\hline & SATIRE-T & 35.572 & & 7.576 & & 2.583 & & 0.407 & & 0.047 & \\
\hline & SATIRE-S & 57.481 & 0.329 & 12.090 & 1.183 & 3.601 & 5.244 & 0.552 & 44.874 & 0.068 & 42.130 \\
\hline & CMIP6 & 53.943 & 0.309 & 11.741 & 1.149 & 3.431 & 4.997 & 0.453 & 36.855 & 0.068 & 41.856 \\
\hline \multirow{5}{*}{$\begin{array}{l}\text { Cycle } 22 \\
\text { descent } \\
\text { Max:Nov.1989 } \\
\text { Min:Jun.1996 }\end{array}$} & 14 & 49.420 & & & & 3.0 & & 0.511 & & 0.102 & \\
\hline & NRLSSI2 & 53.857 & 0.279 & 12.175 & 1.078 & 3.485 & 4.594 & 0.384 & 28.299 & 0.075 & 42.076 \\
\hline & SATIRE-T & 46.024 & 0.233 & 9.820 & 0.848 & 3.363 & 4.321 & 0.557 & 39.972 & 0.085 & 46.134 \\
\hline & SATIRE-S & 64.121 & 0.304 & 13.546 & 1.096 & 4.044 & 4.869 & 0.629 & & 0.085 & 43.436 \\
\hline & CMIP6 & 59.005 & 0.291 & 12.862 & 1.083 & 3.767 & 4.722 & 0.507 & 35.494 & 0.080 & 42.850 \\
\hline \multirow{5}{*}{$\begin{array}{l}\text { Cycle } 23 \\
\text { ascent } \\
\text { Max:Mar.2000 } \\
\text { Min:Jun.1996 }\end{array}$} & LSSI1 & 40.457 & 0.200 & 10.116 & 1.223 & 3.177 & 5.716 & 0.237 & 772 & 0.070 & 53.692 \\
\hline & NRLSSI2 & 42.445 & 0.360 & 9.594 & 1.388 & 2.730 & 5.879 & 0.274 & 32.971 & 0.040 & 36.438 \\
\hline & SATIRE-T & 27.988 & 0.383 & 5.989 & 1.399 & 2.066 & 7.180 & 0.312 & 60.660 & 0.025 & 37.320 \\
\hline & SATIRE-S & 49.546 & 0.473 & 10.446 & 1.704 & 3.041 & 7.380 & 0.439 & 59.580 & 0.034 & 35.442 \\
\hline & CMIP6 & 46.007 & 0.407 & 10.021 & 1.514 & 2.887 & 6.492 & 0.357 & 44.849 & 0.037 & 35.713 \\
\hline
\end{tabular}


Table S2: Solar cycle SSI variations for the average of the five Solar Cycle amplitudes of Table S1 relative to ATLAS3 ( $\Delta S S I)$ in $\%$ and relative contribution of SSI changes to the TSI change $\left(\frac{\Delta S S I}{\Delta T S I}\right)$ in $\%$ for the Lyman- $\alpha(121.5 \mathrm{~nm})$, Far-UV (121-200 nm), Herzberg continuum/Hartley bands (201-242 nm), Hartley-/Huggings-bands (243-380 nm) and visible (381-780 nm) spectral ranges. $\pm 95 \%$ CI indicates the confidence interval.

\begin{tabular}{|c|c|c|c|c|c|c|c|c|c|c|c|}
\hline Time period & SSI dataset & \multicolumn{2}{|c|}{$\begin{array}{c}121.5 \mathrm{~nm} \\
\Delta S S I \frac{\Delta S S I}{\Delta T S I}\end{array}$} & \multicolumn{2}{|c|}{$\begin{array}{c}121-200 \mathrm{~nm} \\
\Delta S S I \frac{\Delta S S I}{\Delta T S I}\end{array}$} & \multicolumn{2}{|c|}{$\begin{array}{l}201-242 \mathrm{~nm} \\
\Delta S S I \frac{\Delta S S I}{\Delta T S I}\end{array}$} & \multicolumn{2}{|c|}{$\begin{array}{l}243-380 \mathrm{~nm} \\
\Delta S S I \frac{\Delta S S I}{\Delta T S I}\end{array}$} & \multicolumn{2}{|c|}{$\begin{array}{r}381-780 \mathrm{~nm} \\
\Delta S S I \frac{\Delta S S I}{\Delta T S I}\end{array}$} \\
\hline \multirow{2}{*}{ Average } & NRLSSI1 & 46.101 & 0.253 & 11.514 & 1.081 & 3.628 & 5.069 & 0.289 & 22.411 & 0.094 & 55.018 \\
\hline & $\pm 95 \% \mathrm{CI}$ & 4.576 & 0.029 & 1.138 & 0.127 & 0.363 & 0.578 & 0.039 & 1.228 & 0.018 & 0.871 \\
\hline \multirow{8}{*}{ Cycle } & NRLSSI2 & 50.989 & 0.286 & 11.527 & 1.105 & 3.300 & 4.705 & 0.364 & 28.681 & 0.071 & 41.474 \\
\hline & $\pm 95 \% \mathrm{CI}$ & 5.931 & 0.053 & 1.341 & 0.203 & 0.393 & 0.843 & 0.061 & 3.098 & 0.023 & 3.533 \\
\hline & SATIRE-T & 40.238 & 0.272 & 8.584 & 0.990 & 2.938 & 5.050 & 0.479 & 45.217 & 0.067 & 43.786 \\
\hline & $\pm 95 \% \mathrm{CI}$ & 9.150 & 0.079 & 1.945 & 0.291 & 0.660 & 1.504 & 0.129 & 10.883 & 0.035 & 4.617 \\
\hline & SATIRE-S & 55.663 & 0.344 & 11.725 & 1.236 & 3.486 & 5.457 & 0.535 & 46.453 & 0.065 & 41.335 \\
\hline & $\pm 95 \% \mathrm{CI}$ & 7.910 & 0.084 & 1.685 & 0.302 & 0.510 & 1.241 & 0.085 & 8.435 & 0.021 & 3.832 \\
\hline & CMIP6 & 53.340 & 0.311 & 11.627 & 1.159 & 3.395 & 5.029 & 0.450 & 36.944 & 0.069 & 41.468 \\
\hline & $\pm 95 \% \mathrm{CI}$ & 6.249 & 0.065 & 1.362 & 0.239 & 0.411 & 0.988 & 0.065 & 5.392 & 0.020 & 3.792 \\
\hline
\end{tabular}



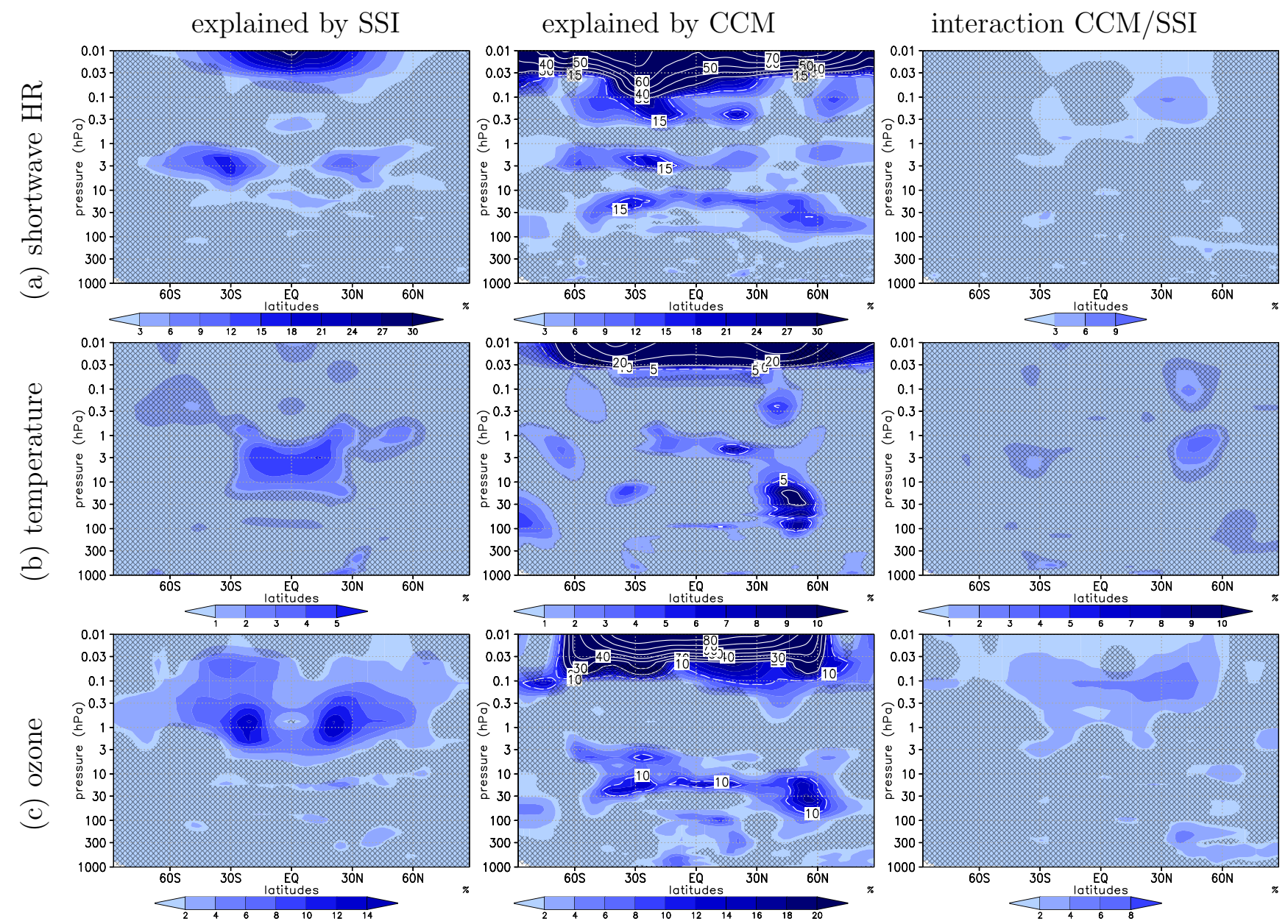

Figure S1: As Figure 4 in the main text, but without the simulations using the SATIRE-T SSI data set. Left column: Percentage of signal variance (square of white contours of left figures) explained by systematic differences between forcing SSI data sets ( $\mathrm{R}_{a, B}$ Equation A11, blue shading). The white contours indicate levels of explained variance larger than the range of shading. Middle column: as left column but for systematic differences between CCMs $\left(\mathrm{R}_{a, A}\right.$ Equation A11). Right column: as left column but for signal variance explained by the interaction of the CCM and the SSI data set treatments $\left(\mathrm{R}_{a, A B}\right.$ Equation A12). The grey hatching masks areas where the ratio of explained variance does not pass a test for statistical significance $(\mathrm{p}>5 \%)$. 

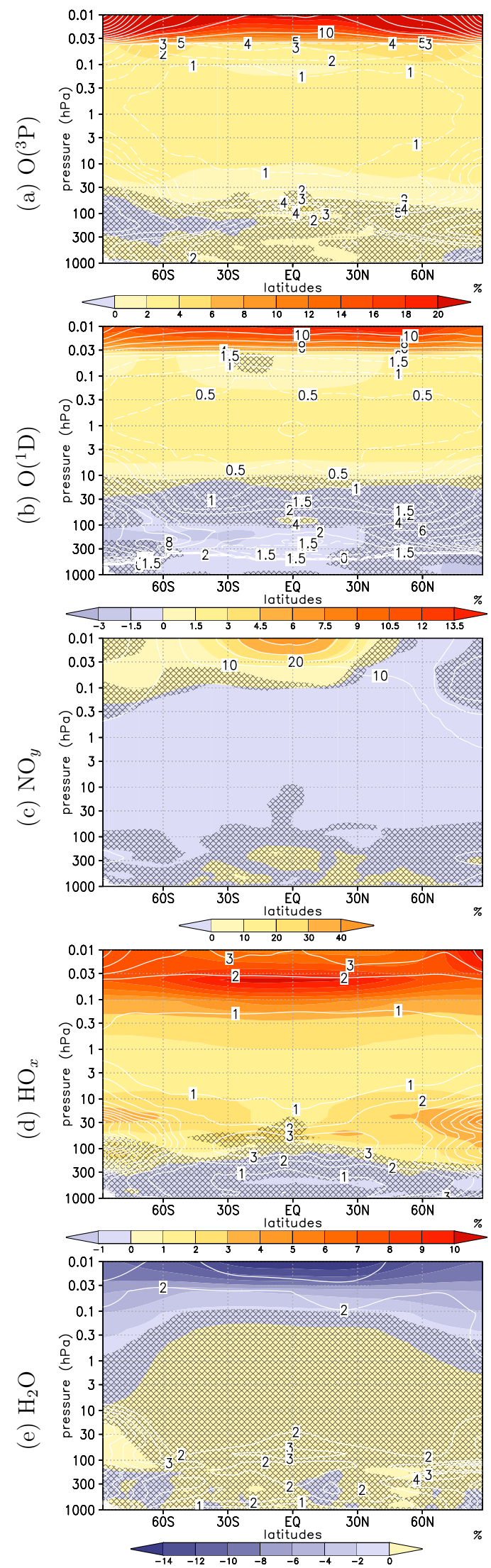

Figure S2: Annual mean 11-year solar cycle response (shaded) and signal variance (white contours) in terms of the solar response annual standard deviation for (a) $\mathrm{O}\left({ }^{3} \mathrm{P}\right)$, (b) $\mathrm{O}\left({ }^{1} \mathrm{D}\right)$, (c) $\mathrm{NO}_{\mathrm{y}}$, (d) $\mathrm{HO}_{\mathrm{x}}$, and (e) $\mathrm{H}_{2} \mathrm{O}$ mixing ratios. Solar signal derived as ensemble mean over both models and all SSI data sets; solar minimum SSI based on ATLAS3 reference state. The grey hatching masks areas where the solar cycle response does not pass a test for statistical significance $(\mathrm{p}>5 \%)$. 
explained by SSI
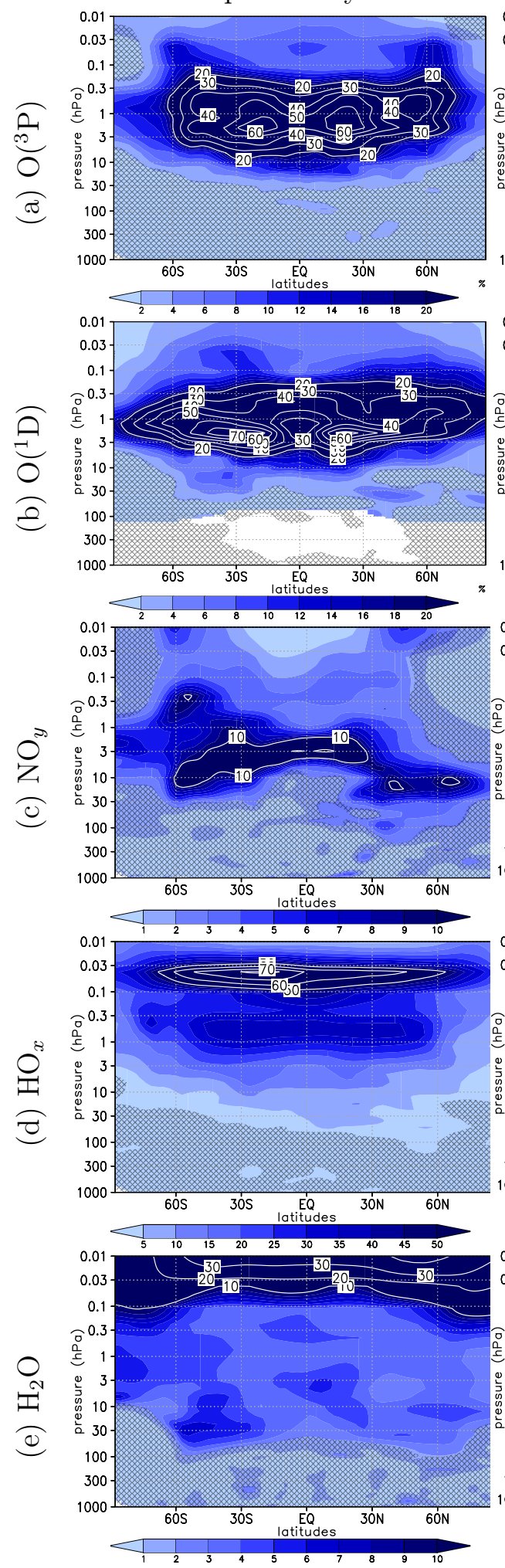

explained by CCM
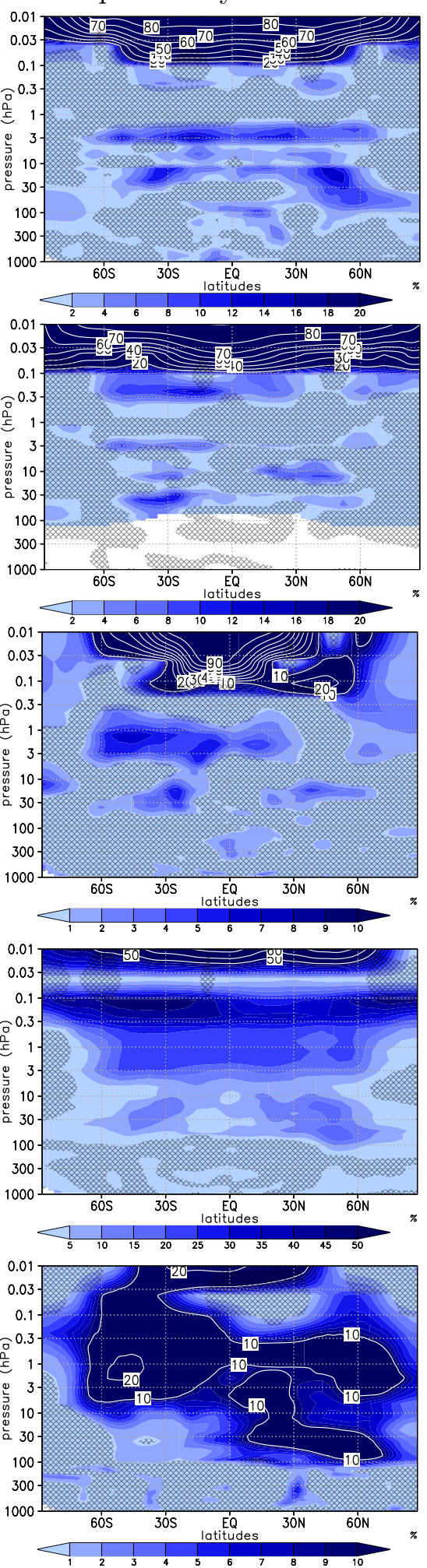

interaction $\mathrm{CCM} / \mathrm{SSI}$
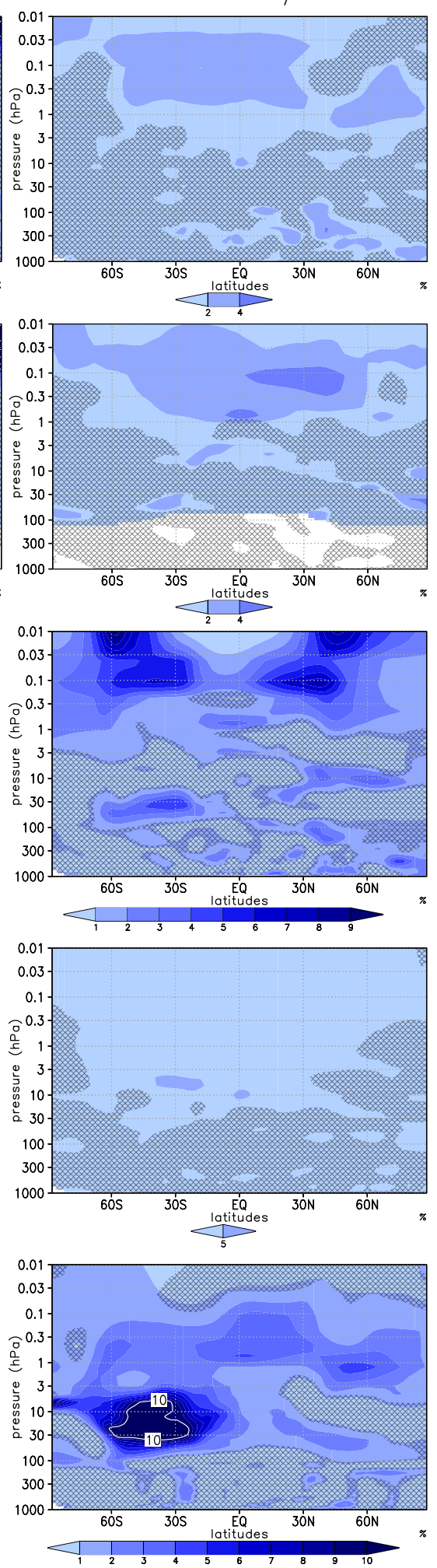

Figure S3: Percentage of solar cycle response variance (square of white contours in Figure 2) explained for (a) $O\left({ }^{3} \mathrm{P}\right)$, (b) $\mathrm{O}\left({ }^{1} \mathrm{D}\right)$, (c) $\mathrm{NO}_{\mathrm{y}}$, (d) $\mathrm{HO}_{\mathrm{x}}$, and (e) $\mathrm{H}_{2} \mathrm{O}$ mixing ratios. Left column: explained by systematic differences between forcing SSI data sets ( $\mathrm{R}_{a, B}$ Equation A11, blue shading). The white contours indicate levels of explained variance larger than the range of shading. Middle column: as left column but for systematic differences between CCMs ( $\mathrm{R}_{a, A}$ Equation A11). Right column: as left column but for signal variance explained by the interaction of the CCM and the SSI data set treatments ( $\mathrm{R}_{a, A B}$ Equation A12). The grey hatching masks areas where the ratio of explained variance does not pass a test for statistical significance $(\mathrm{p}>5 \%)$. 


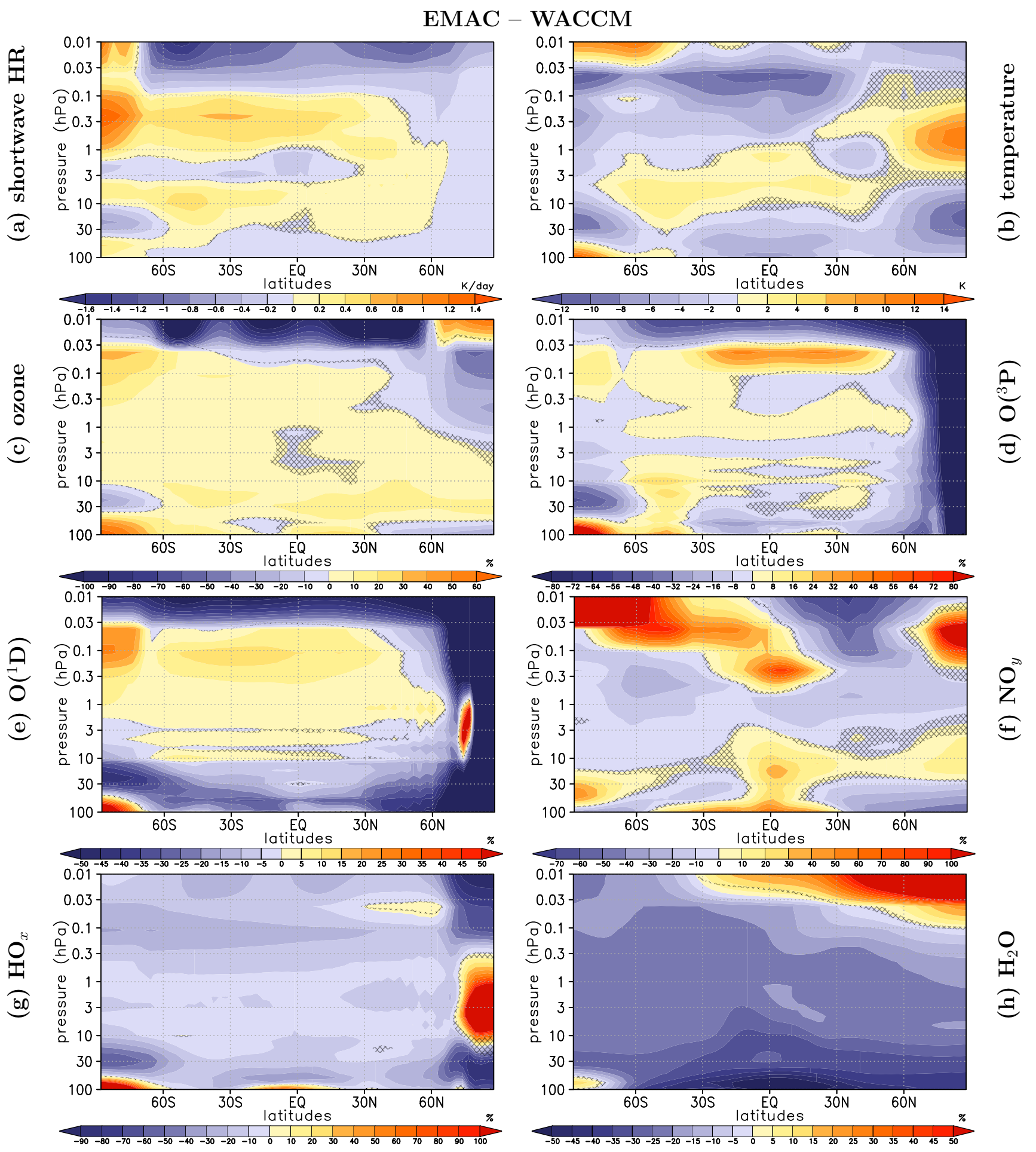

Figure S4: January mean differences for EMAC (ensemble mean) minus WACCM (ensemble mean) (shaded) of (a) shortwave heating rates, (b) temperature, (c) ozone mixing ratios, (d) atomic oxygen $\left(\mathrm{O}\left({ }^{3} \mathrm{P}\right)\right)$, (e) $\mathrm{HO}_{\mathrm{x}}$, and (f) $\mathrm{NO}_{\mathrm{y}}$. The ensemble mean for both CCMs consists of the solar minimum reference simulation (included 5 times in the ensemble mean) and the 5 simulations for the solar maximum. Grey hatching masks areas where differences does not pass a test for statistical significance $(\mathrm{p}>5 \%)$. 


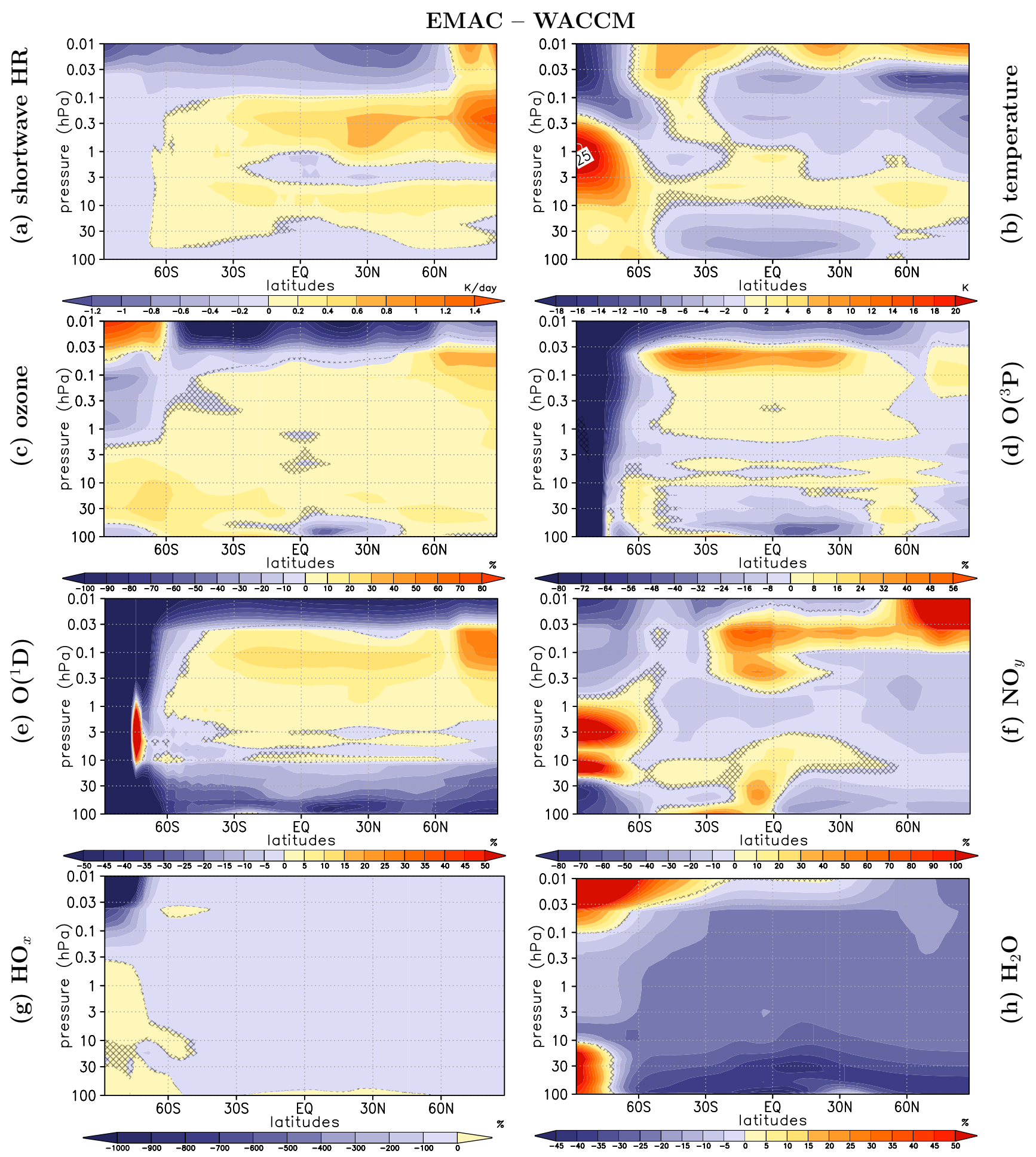

Figure S5: July mean differences for EMAC (ensemble mean) minus WACCM (ensemble mean) (shaded) of (a) shortwave heating rates, (b) temperature, (c) ozone mixing ratios, (d) atomic oxygen $\left(\mathrm{O}\left({ }^{3} \mathrm{P}\right)\right.$ ), (e) $\mathrm{HO}_{\mathrm{x}}$, and (f) $\mathrm{NO}_{\mathrm{y}}$. The ensemble mean for both CCMs consists of the solar minimum reference simulation (included 5 times in the ensemble mean) and the 5 simulations for the solar maximum. Grey hatching masks areas where differences does not pass a test for statistical significance (p $>5 \%)$. 
Table S3: Correlation of polar region $\left(70^{\circ} \mathrm{N}-90^{\circ} \mathrm{N}\right)$ anomalies (solar maximum - solar minimum) of total column ozone (TCO) and the layer thickness from 100 to $10 \mathrm{hPa}$. TCO change in DU per $100 \mathrm{~m}$ geopotential height change and the $95 \%$ confidence interval.

\begin{tabular}{|c|c|c|c|c|c|}
\hline \multirow[b]{2}{*}{ Hemisphere } & \multirow[b]{2}{*}{ Season } & \multicolumn{2}{|c|}{ EMAC } & \multicolumn{2}{|c|}{ WACCM } \\
\hline & & Correlation & $\Delta \mathrm{TCO} / 100 \mathrm{~m}$ & Correlation & $\Delta \mathrm{TCO} / 100 \mathrm{~m}$ \\
\hline & & \multicolumn{4}{|c|}{ CMIP6 } \\
\hline \multirow[t]{2}{*}{ NH } & Anm & 0.82 & $6.08 \pm 0.37$ & 0.68 & $6.11 \pm 0.57$ \\
\hline & JFM & 0.89 & $6.79 \pm 1.05$ & 0.70 & $5.49 \pm 1.72$ \\
\hline \multirow{3}{*}{$\mathrm{SH}$} & Anm & 0.81 & $7.49 \pm 0.47$ & 0.77 & $6.06 \pm 0.43$ \\
\hline & SON & 0.92 & $8.95 \pm 1.14$ & 0.95 & $5.74 \pm 0.61$ \\
\hline & & \multicolumn{4}{|c|}{ SATIRE-T } \\
\hline \multirow[t]{2}{*}{$\mathrm{NH}$} & Anm & 0.81 & $5.91 \pm 0.37$ & 0.69 & $6.03 \pm 0.55$ \\
\hline & JFM & 0.88 & $6.14 \pm 1.02$ & 0.87 & $6.29 \pm 1.12$ \\
\hline \multirow[t]{3}{*}{$\mathrm{SH}$} & Anm & 0.82 & $8.02 \pm 0.48$ & 0.76 & $5.38 \pm 0.40$ \\
\hline & SON & 0.95 & $9.79 \pm 1.02$ & 0.91 & $5.13 \pm 0.73$ \\
\hline & & \multicolumn{4}{|c|}{ SATIRE-S } \\
\hline \multirow[t]{2}{*}{ NH } & Anm & 0.83 & $6.20 \pm 0.36$ & 0.71 & $5.91 \pm 0.51$ \\
\hline & JFM & 0.89 & $6.75 \pm 1.05$ & 0.76 & $6.31 \pm 1.67$ \\
\hline \multirow[t]{3}{*}{ SH } & Anm & 0.81 & $7.72 \pm 0.48$ & 0.69 & $5.09 \pm 0.46$ \\
\hline & SON & 0.95 & $9.30 \pm 0.89$ & 0.88 & $4.66 \pm 0.78$ \\
\hline & & \multicolumn{4}{|c|}{ NRLSSI1 } \\
\hline \multirow[t]{2}{*}{ NH } & Anm & 0.84 & $5.95 \pm 0.34$ & 0.70 & $6.25 \pm 0.55$ \\
\hline & JFM & 0.90 & $6.00 \pm 0.92$ & 0.81 & $6.96 \pm 1.55$ \\
\hline \multirow[t]{3}{*}{$\mathrm{SH}$} & Anm & 0.82 & $7.26 \pm 0.44$ & 0.76 & $5.94 \pm 0.44$ \\
\hline & SON & 0.91 & $8.95 \pm 1.24$ & 0.92 & $5.35 \pm 0.70$ \\
\hline & & \multicolumn{4}{|c|}{ NRLSSI2 } \\
\hline \multirow[t]{2}{*}{ NH } & Anm & 0.82 & $6.39 \pm 0.38$ & 0.66 & $5.84 \pm 0.57$ \\
\hline & JFM & 0.94 & $6.48 \pm 0.75$ & 0.80 & $6.81 \pm 1.58$ \\
\hline \multirow[t]{2}{*}{$\mathrm{SH}$} & Anm & 0.83 & $7.81 \pm 0.45$ & 0.76 & $5.54 \pm 0.41$ \\
\hline & SON & 0.94 & $9.63 \pm 1.10$ & 0.94 & $5.42 \pm 0.61$ \\
\hline
\end{tabular}

